\title{
Outcomes of vaginal hysterectomy and constricting colporrhaphy with concurrent levator myorrhaphy and high perineorrhaphy in women older than 75 years of age
}

\author{
This article was published in the following Dove Press journal: \\ Clinical Interventions in Aging \\ 24 June 2015 \\ Number of times this article has been viewed
}

\section{Emrah Töz \\ Aykut Özcan \\ Nesin Apaydın \\ İbrahim Uyar \\ Betül Kocakaya \\ Gülin Okay}

Department of Gynecology and Obstetrics, Tepecik Education and Research Hospital, İzmir, Turkey
Correspondence: Emrah Töz Department of Gynecology and Obstetrics, Tepecik Education and Research Hospital, Gaziler cad, No 468, İzmir 35330, Turkey

Tel +905052595629

Fax+90232261 735।

Email emrahtoz79@gmail.com
Objectives: We performed constricting anterior and posterior colporrhaphy, levator myorrhaphy, and high perineorrhaphy with concurrent hysterectomy, and investigated the intraoperative complications, and short-term outcomes of these constricting procedures in patients aged 75 years or older.

Methods: We searched our hospital database for cases, between January 2011 and January 2014, of women aged over 75 years who underwent surgery for pelvic organ prolapse of stage 2 or higher, via vaginal hysterectomy, constricting anterior and posterior colporrhaphy, levator myorrhaphy, and high perineorrhaphy, with or without treatment of urinary incontinence. All volunteers were evaluated via pelvic examination using the pelvic organ prolapse quantification system, the modified Decision Regret Scale-Pelvic Floor Disorders form, the Satisfaction Decision Scale-Pelvic Floor Disorders form, and the Pelvic Floor Distress Inventory form.

Results: Fifty-four patients were included in the study. The mean follow-up time was 24.4 months after constricting surgery (range: 8-44 months). There were four cases (7\%) of de novo urge incontinence (the symptoms resolved upon prescription of anticholinergic medication). Two patients developed de novo stress urinary incontinence after the procedure and were treated via transobturator sling surgery using Safyre $\mathrm{T}^{\circledR}$ polypropylene monofilament slings. No anatomical or subjective recurrence of prolapse was noted during the follow-up period. No patient required additional surgery for recurrence of prolapse.

Conclusion: Constricting anterior and posterior colporrhaphy, levator myorrhaphy, and high perineorrhaphy with concurrent hysterectomy is a feasible, safe, and effective surgical option in elderly patients at low anesthesiological risk. The decision to perform an incontinence procedure should be individualized based on preoperative findings after prolapse reduction.

Keywords: older women, pelvic organ prolapse, constrictive surgical procedures, urinary incontinence

\section{Introduction}

Pelvic organ prolapse (POP) is a major health issue for women, affecting quality-oflife and requiring costly surgery., ${ }^{1,2} \mathrm{POP}$ is an age-related disorder, and the prevalence increases as a population ages. Women who attain the age of 80 years have a greater than $10 \%$ risk of requiring surgery to treat POP. ${ }^{3}$ Treatment of genital prolapse in elderly women is challenging and almost always depends on the medical condition and sexual activity level of the patient. Although pessaries are offered as first-line therapy to such patients, ${ }^{4,5}$ surgery may be indicated for patients who have failed or do not desire the lifelong use of a pessary. 
Various surgical techniques using laparotomy, laparoscopy, and vaginal surgery, including colporrhaphy (with or without mesh placement), sacrospinous ligament fixation (SSLF), abdominal sacrocolpopexy (ASC), high sacrouterine ligament fixation, prolapse kit placement, colpocleisis, and constricting surgical procedures, have been utilized. These procedures differ in terms of both success and complication rates. ASC has an $80 \%-85 \%$ long-term success rate, while SSLF has a $70 \%-75 \%$ long-term success rate. ${ }^{6,7}$ Prolapse kits (Gynecare Prolift ${ }^{\circledR}$; Ethicon, Inc., Somerville, NJ, USA) have a success rate of $96.5 \% .{ }^{8}$ However, all procedures may cause major complications, such as bladder injury, hemorrhage, wound dehiscence, rectal injury, and postoperative vault infection. ASC has disadvantages, including a long operative time, a long recovery time, possible injury to pelvic structures, hemorrhage, and ileus. SSLF is a technically challenging approach - the surgical site is deep and narrow. SSLF also has disadvantages, including the possibilities of hemorrhage, infection, and nerve damage. Prolift kits can have adverse events, such as bladder injury and mesh erosion.

On the other hand, obliterative and constrictive surgical procedures have lower levels of complications, a low risk of perioperative morbidity, and an extremely low risk of prolapse recurrence. Patients who are not interested in a functional vagina because they are not sexually active and have no desire to be so active in the future, are the best candidates for obliterative or constrictive vaginal surgery. Various techniques have been described, but LeFort colpocleisis is the preferred procedure for obliterative surgery. ${ }^{9-11}$ Although colpocleisis with uterine preservation is effective, drawbacks are apparent. First, it precludes future evaluation of the cervix and uterus. Other drawbacks include late vaginal bleeding, ureteric occlusion or injury as a result of kinking in the distal ureter, a risk of gynecological cancer, vaginal discharge, and an uncomfortable feeling of fullness in the vagina. ${ }^{12,13}$ Constricting surgical procedures are alternatives avoiding these drawbacks. The goal is to narrow the vaginal tube to close the defect.

\section{Objectives}

We performed constricting anterior and posterior colporrhaphy, levator myorrhaphy, and high perineorrhaphy with concurrent hysterectomy, and investigated the feasibility, intraoperative complications, and short-term follow-up results of these constricting procedures in 54 patients aged 75 years or older.

\section{Material and methods}

This study was performed at the Obstetrics and Gynecology Department at Tepecik Training and Research Hospital.
The study was approved by the institutional review board of Tepecik Education and Research Hospital and all participants provided informed consent. The hospital database was searched for cases treated between January 2011 and January 2014; we sought women over the age of 75 years who underwent surgery for pelvic floor disorders, with or without incontinence surgery. In total, 202 such women were identified. With the increase in life expectancy, an age of 75 years has been used as a cutoff for "elderly" in several studies. ${ }^{14,15} \mathrm{We}$ used the same cutoff.

Our study inclusion criteria were an age greater than 75 years and surgery for POP stage 2 or higher (Figure 1), with vaginal hysterectomy, constricting anterior and posterior colporrhaphy, levator myorrhaphy, and a high perineorrhaphy, with or without a urinary incontinence procedure. A total of 123 patients who underwent other types of POP surgery were excluded. Patients were contacted via telephone and invited to participate in the study. A flow chart of the study design is presented in Figure 2.

Demographic characteristics, including age, parity, mode of delivery, and the presence/absence of hypertension and diabetes, were recorded. All patients provided detailed histories and underwent complete gynecological examinations. Prolapse assessment was performed by a specialist, using the POP quantification (POP-Q) system. ${ }^{16}$ Objective recurrence

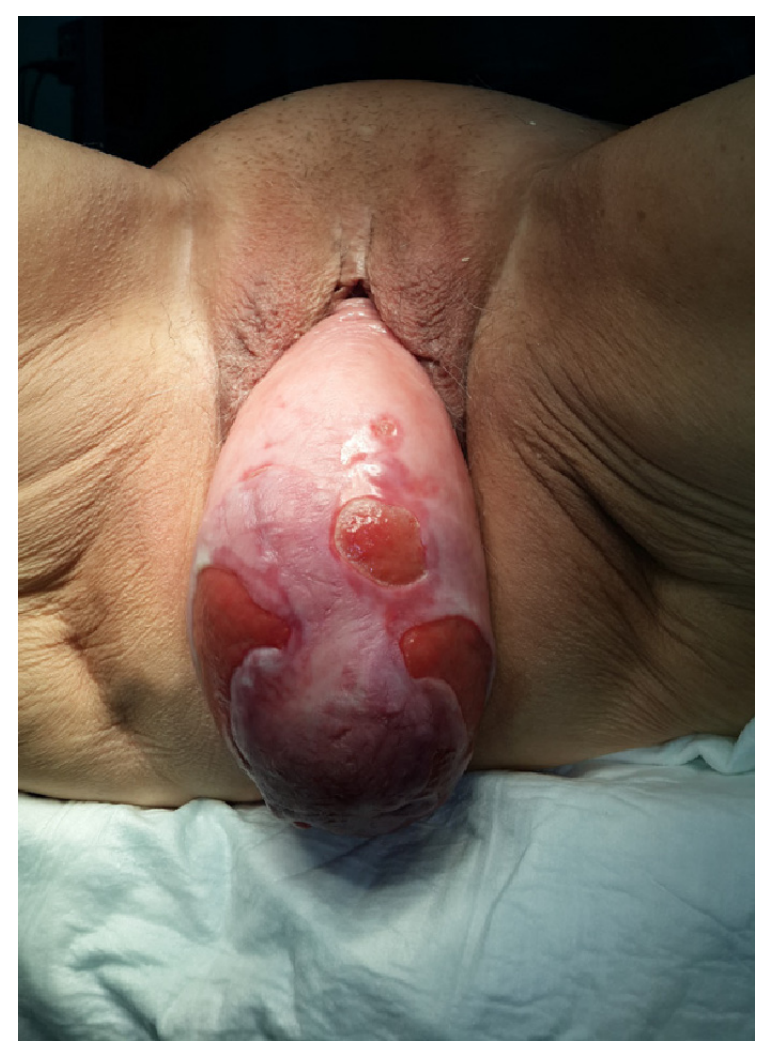

Figure I A patient with stage 4 pelvic organ prolapse. 


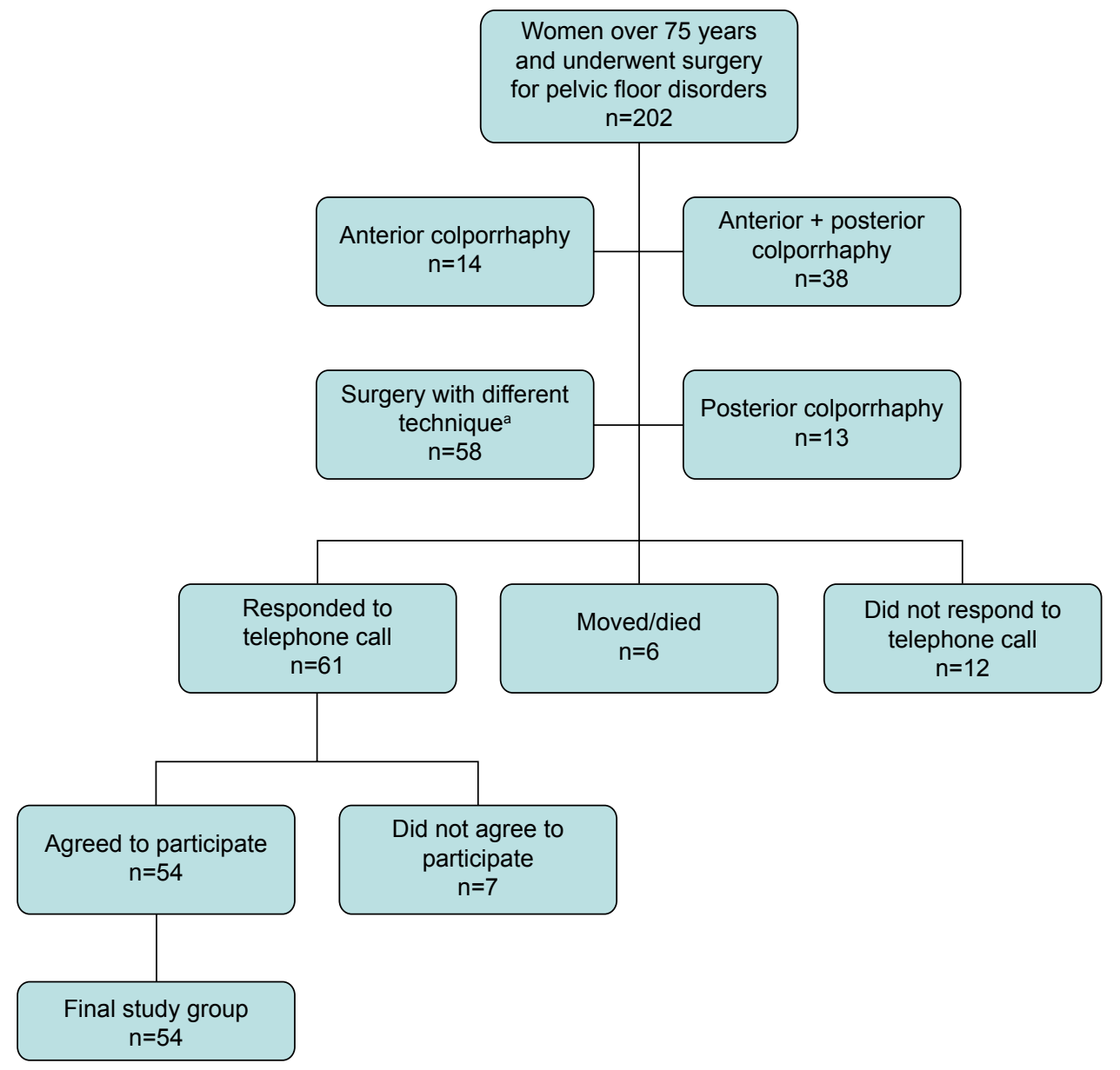

Figure 2 Flowchart of the study.

Note: aSacrospinous fixation, sacrocolpopexy, or high uterosacral ligament suspension with or without hysterectomy

was defined as stage 2 or higher POP. Subjective recurrence was defined as a perception of prolapse (bulge symptoms) by the patient. Regret and satisfaction were assessed using the Decision Regret Scale-Pelvic Floor Disorders (DRS-PFD) and Satisfaction Decision Scale-Pelvic Floor Disorders (SDS-PFD) forms. ${ }^{17}$ The DRS-PFD is a five-item questionnaire with a five-point response scale. Scores are given by using the mean of these items (range 1-5). Higher scores indicate a higher degree of decision regret. The SDS-PFD is a six-item questionnaire with a five-point response scale. Results are given as the mean of these items (range 1-5). Higher scores indicate higher overall satisfaction. The Pelvic Floor Distress Inventory (PFDI) was used to assess quality of life outcomes. ${ }^{18}$ PFDI has three subscales: the Urinary Distress Inventory, the Colorectal-Anal Distress Inventory, and the Pelvic Organ Prolapse Distress Inventory. Patient answers are ranked on a Likert scale, with $1=$ not at all, $2=$ somewhat, $3=$ moderately, and $4=$ quite a bit. "No" answers receive a score of 0 . The mean value of all questions answered is then multiplied by 25 for the actual score (range 0-100).
The higher the score, the greater the perceived impact that pelvic floor dysfunction has on a patient's life.

Statistical analyses were conducted using SPSS 18.0 (IBM Corp., Armonk, NY, USA). Descriptive statistics were used to characterize all patients. Results were presented as mean \pm standard deviation (SD) or as median (interquartile range).

\section{Operative technique}

Preoperatively, all patients underwent a stress test in the standing position, at a bladder volume of $300 \mathrm{~mL}$, with the pessary placed and the prolapse reduced. The results of the cough stress test were recorded as positive if urine loss occurred with a cough, or as negative if no urine loss was noted. All patients received vaginal estrogen therapy for at least 1 month before surgery. They received an intravenous first-generation cephalosporin 1 hour before surgery and underwent a prophylactic antiseptic vaginal wash with iodine-containing soap before surgery. All surgeries were performed under regional anesthesia. 
The procedure commenced with hysterectomy using "the ten-step vaginal hysterectomy" technique. ${ }^{19}$ The vaginal cuff was left open until the anterior colporrhaphy was concluded. After hysterectomy, the anterior midline of the vaginal cuff was used as the starting point for anterior vaginal wall incision. A full-length and full-width dissection of the anterior vaginal wall from the underlying musculoconnective tissue was performed. After dissection of the vaginal epithelium from the underlying pubocervical fascia, a Vicryl \#2 suture was placed on the fascias, using a continuous circular clockwise motion. The floor of the bladder was next pushed up as the purse-string suture was tied. Excess vaginal epithelium was excised, and the paravaginal connective tissue and vaginal epithelium were approximated in the midline, using a delayed absorbable suture. After the anterior colporrhaphy, the vaginal cuff was closed with three interrupted 0-Dexon sutures. Ovarian ligaments, the round ligaments, and the cardinal and uterosacral ligaments were attached to the vaginal vault to support the vaginal apex. Next, two Allis clamps were placed at the 4- and 8-o'clock positions of the hymenal ring. A horizontal incision was made in the vaginal epithelium, from clamp to clamp. The incision was then extended upwards from the midline to the vaginal cuff. The goal of vaginal incision was to allow separation of epithelium from the underlying connective tissue. The plane of separation was continued as far laterally as necessary, in either direction, toward the pelvic sidewall to expose the puborectalis muscle, the bulbocavernosus muscle, and the perineal membrane. Excess vaginal epithelium was excised and the puborectalis muscle, the bulbocavernosus muscle, and the vaginal epithelium approximated in the midline, using a delayed absorbable suture. The perineal body was then approximated. Finally, the vaginal introital epithelium was approximated with an absorbable running suture, rendering the resultant genital hiatus about $1 \mathrm{~cm}$ in length (Figure 3).

A concomitant transobturator sling operation, using a Safyre $T^{\circledR}$ (Promedon, Cordoba, Argentina) polypropylene monofilament sling (placed through a separate vaginal incision) was performed in women for whom the stress test was positive. After surgery, saline-soaked gauze was packed into the vagina, and a urethral catheter inserted. Routine cystoscopy was not performed.

\section{Results}

A total of 79 patients were operated. Of these 79 patients, 12 did not respond to phone calls, six patients moved or died, and seven patients did not agree to participate in the study. There was no significant difference between the study group

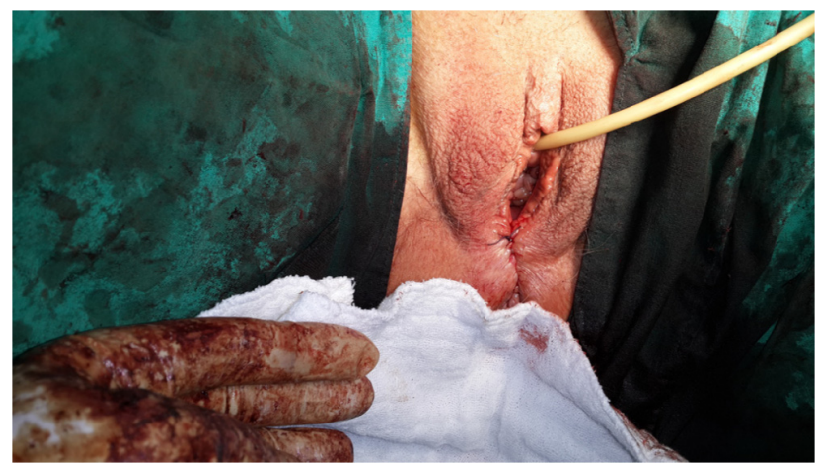

Figure 3 The same patient of Figure I after the vaginal introital epithelium was approximated with an absorbable running suture, leaving the resultant genital hiatus of about $\mathrm{I} \mathrm{cm}$ in length.

and the nonresponders/nonagreement cohorts in terms of age, body mass index, parity, comorbidities, and stage of prolapse (data not shown).

A total of 54 patients were included in the study. The patients' mean (SD) age was 79.7 (2.6) years, the mean (SD) body mass index was $29.14(3.16) \mathrm{kg} / \mathrm{m}^{2}$, and the mean (SD) parity was 3.51 (1.55). All patients had stage 2 or greater prolapse. Of the 54 patients, 17 had preoperative occult urinary incontinence diagnosed upon stress testing using a pessary. These patients were fitted with a midurethral Safyre $T^{\circledR}$ polypropylene monofilament sling. The clinical and demographic characteristics of the patients are summarized in Table 1.

The mean (SD) operating time was 129.6 (11.9) minutes. Intraoperative complications occurred in only one patient: this was a bladder injury that was identified and repaired intraoperatively (Table 2). Three patients fitted with

Table I Clinical and demographic characteristics of the patients

\begin{tabular}{ll}
\hline Characteristics & Study group $(\mathbf{n = 5 4})$ \\
\hline Age (years) & $79.7 \pm 2.6(95 \% \mathrm{Cl}, 79.04-80.5 \mathrm{I})$ \\
Body mass index $\left(\mathrm{kg} / \mathrm{m}^{2}\right)$ & $29.14 \pm 3.16(95 \% \mathrm{Cl}, 28.28-30.0 \mathrm{I})$ \\
Parity & $3.5 \mathrm{I} \pm \mathrm{I} .55(95 \% \mathrm{Cl}, 3.09-3.94)$ \\
Smoking status & $3(6 \%)$ \\
Hypertension & $19(35 \%)$ \\
Diabetes mellitus & $12(22 \%)$ \\
Preoperative stage of prolapse & \\
$\quad$ II & $15(28 \%)$ \\
$\quad$ III & $28(52 \%)$ \\
$\quad$ IV & $\mathrm{II}(20 \%)$ \\
Operation time (minute) & $129.6 \pm \mathrm{II} .9(95 \% \mathrm{Cl}, \mathrm{I26.38-132.87)}$ \\
Associated anti-incontinence & $\mathrm{I} 7(3 \mathrm{I} \%)$ \\
surgery & \\
Hospital stay (days) & $3.48 \pm \mathrm{I} .02(95 \% \mathrm{Cl}, 3.20-3.76)$ \\
Follow-up times (months) & $24.42 \pm 9.8 \mathrm{I}(95 \% \mathrm{Cl}, 2 \mathrm{I} .7-27 . \mathrm{I})$ \\
\hline
\end{tabular}

Note: Data are expressed as mean \pm standard deviation $(95 \% \mathrm{Cl})$ or $\mathrm{n}(\%)$. Abbreviation: $\mathrm{Cl}$, confidence interval. 
Table 2 Intraoperative and postoperative complications

\begin{tabular}{lc}
\hline & $\mathbf{n ~ ( \% )}$ \\
\hline Intraoperative complications & \\
$\quad$ Bladder injury & I (2\%) \\
Postoperative complications & \\
$\quad$ Voiding difficulty & $3(6 \%)$ \\
De novo urge incontinence & $4(7 \%)$ \\
De novo stress urinary incontinence & $2(4 \%)$ \\
\hline
\end{tabular}

midurethral slings had postoperative voiding difficulties, which spontaneously resolved within 3 days. The mean (SD) hospital stay was 3.48 (1.02) days.

The mean follow-up time was 24.4 months after constricting surgery (range: 8 to 44 months). The mean PFDI score was 29.6 , suggesting a good quality of life. The mean DRS-PFD score was 1.23 , showing very little regret. The mean SDS-PFD score was 4.82, suggesting a high satisfaction (Table 3). All regrets and or dissatisfactions were attributable to urinary complaints. There were four cases $(7 \%)$ of de novo urge incontinence (the symptoms resolved with anticholinergic medication). No stress urinary incontinence developed in patients treated with concomitant incontinence surgery. Two patients developed de novo stress urinary incontinence after the procedure and were given midurethral sling surgery. No recurrence of incontinence was seen during follow up.

No anatomical (no prolapse beyond hymen) or subjective recurrence of the prolapse was noted during follow up. No patients required additional surgery for recurrence of prolapse.

\section{Discussion}

We found that treatment of advanced prolapse using a constricting technique in elderly women is a feasible, safe, and effective surgical option that may represent an alternative to colpocleisis and other techniques that install mesh or graft material. The complication rates were low, but the operation time and hospital stay rather long in our study. Only one intraoperative complication occurred, and no patient developed prolapse recurrence during follow up. The low complication and high success rates are similar to those of other studies exploring the colpocleisis procedure and new sling techniques. Lu et al ${ }^{12}$ reported on 63 elderly POP patients of stages 3 or 4 who underwent total or partial colpocleisis. No intraoperative injury and no recurrence were observed. Similarly, Zebede et $\mathrm{al}^{10}$ reported a $98.1 \%$ anatomical success rate in elderly women after LeFort colpocleisis. Cho et al ${ }^{20}$ showed that the anatomical success rate was $97.1 \%$ after total mesh repair using the Prolift technique to treat advanced POP in elderly women. Although the complication and success rates were similar to those of obliterative techniques, the mean operating time was longer in our study. ${ }^{12,20}$

The ultimate decision on the approach and procedure when older women require POP should be based on the overall health, physical activity status, and future sexual activity plans of the patient, as well as on surgeon training, skills, and preference. Colpocleisis is less invasive, requires a shorter operative time, and has fewer surgical risks compared with constricting procedures. But colpocleisis also has drawbacks. When the pelvic anatomy limits or prevents colpocleisis, or if women chose hysterectomy after being provided with detailed information, a constricting anterior and posterior colporrhaphy, with levator myorrhaphy and high perineorrhaphy, is an option in elderly patients at low anesthesiological risk. A decision to perform (or not) an incontinence procedure in such patients is difficult and should be individualized. Patients should be preoperatively evaluated for urinary incontinence and bladder function because the morbidity of postoperative stress incontinence must be balanced against the possibility of urinary retention.

Hysterectomy is often suggested and performed at the time of POP repair because most of the common techniques for apical prolapse repair require hysterectomy. In SSLF and ASC, the apex is elevated by affixing the vaginal cuff to a support structure. Uterine-sparing procedures correct apical prolapse by attaching the lower uterus or cervix to a support structure. These techniques are not widely used because most surgeons have not been trained to perform them. Also, the common wisdom has been that retaining the uterus increases

Table 3 Questionnaire outcomes

PFDI

Pelvic Organ Prolapse Distress Inventory scores (range 0-100), median (IQR)

$(0-25) 14.7$

Colorectal-Anal Distress Inventory scores (range 0-I00), median (IQR)

$(0-26.4) 5.1$

Urinary Distress Inventory scores (range 0-I00), median (IQR)

PFDI total scores (range 0-100), median (IQR)

$(3.6-27.1) 14.3$

Decision Regret Scale-Pelvic Floor Disorder scores, mean (SD)

$(9.8-68.3) 29.6$

Satisfaction Decision Scale-Pelvic Floor Disorder scores, mean (SD)

$(0.5 \mathrm{I}) 1.23$

Abbreviations: IQR, interquartile range; PFDI, Pelvic Floor Distress Inventory; SD, standard deviation. 
the risk of recurrent prolapse, although there are no data to support this notion. Hysterectomy eliminates future cervical or intrauterine pathology but causes pelvic neuropathy and disruption of natural support structures. Uterine-sparing surgery has less impact on sexual function and may preserve fertility. In our study group, no patient desired a functional vagina, so we chose to perform hysterectomy. The greatest disadvantage of this surgery is that postoperative coital activities are not possible. Thus, preoperative discussion with the patient is essential. We performed constricting surgery in 79 (39\%) of 202 patients who had no desire to be sexually active. We believe that this high rate of sexual inactivity is attributable to culturally induced religious beliefs in our country, to the effect that sexual activity is for the young. None of our patients reported having had intercourse in the previous 1 year.

We performed conventional anterior vaginal wall repair without mesh placement. It has been reported that recurrent anterior vaginal wall prolapse after conventional repair occurs in more than $30 \%$ of cases. ${ }^{21}$ To improve outcomes, synthetic graft materials have been introduced to reinforce native tissues. It has been reported that anterior colporrhaphy with synthetic graft reinforcement significantly reduces anatomical recurrence, but no difference in symptomatic recurrence was noted, and the mesh erosion rate was high. We preferred to not use mesh because of the high mesh erosion rate, and we performed constricting surgery because we did not expect a high recurrence rate.

The major limitations of our study are as follows: there was no control group that underwent either standard nonconstricting repair or colpocleisis; preoperative questionnaires were not completed; and all patients were of American Society of Anesthesiologists anesthesia risk class 1 or $2 .{ }^{22}$ It may be difficult to perform this procedure in patients of higher anesthesia risk classes. However, the performance of all surgeries by the same surgical team enhanced the validity of our study.

\section{Conclusion}

Our results indicate that constricting anterior and posterior colporrhaphy, levator myorrhaphy, and high perineorrhaphy with concurrent hysterectomy, is a feasible, safe, and effective surgical option in elderly patients at low anesthesiological risk. The decision to perform an incontinence procedure should be individualized based on preoperative findings after prolapse reduction.

Constricting procedures should not be the sole forms of surgery offered for prolapse, being a part of the pelvic surgeon's armamentarium when treatment of severe POP is required.

\section{Disclosure}

The authors report no conflicts of interest in this work.

\section{References}

1. Digesu GA, Chaliha C, Salvatore S, Hutchings A, Khullar V. The relationship of vaginal prolapse severity to symptoms and quality of life. BJOG. 2005;112(7):971-976.

2. Subramanian D, Szwarcensztein K, Mauskopf JA, Slack MC. Rate, type, and cost of pelvic organ prolapse surgery in Germany, France, and England. Eur J Obstet Gynecol Reprod Biol. 2009;144(2):177-181.

3. Luber KM, Boero S, Choe JY. The demographics of pelvic floor disorders: current observations and future projections. Am J Obstet Gynecol. 2001;184(7):1496-1501; discussion 1501-1503.

4. Mutone MF, Terry C, Hale DS, Benson JT. Factors which influence the short-term success of pessary management of pelvic organ prolapse. Am J Obstet Gynecol. 2005;193(1):89-94.

5. Powers K, Lazarou G, Wang A, et al. Pessary use in advanced pelvic organ prolapse. Int Urogynecol J Pelvic Floor Dysfunct. 2006;17(2): 160-164.

6. Nygaard I, Brubaker L, Zyczynski HM, et al. Long-term outcomes following abdominal sacrocolpopexy for pelvic organ prolapse. JAMA. 2013;309(19):2016-2024

7. Morgan DM, Rogers MA, Huebner M, Wei JT, Delancey JO. Heterogeneity in anatomic outcome of sacrospinous ligament fixation for prolapse: a systematic review. Obstet Gynecol. 2007;109(6):1424-1433.

8. Hong MK, Chu TY, Wei YC, Ding DC. High success rate and considerable adverse events of pelvic prolapse surgery with Prolift: a single center experience. Taiwan J Obstet Gynecol. 2013;52(3):389-394.

9. Pizarro-Berdichevsky J, Galleguillos G, Cuevas R, et al. Labhardt's colpoperineocleisis: subjective results of an alternative treatment for genital prolapse in patients who are not sexually active - 2-year follow-up. Int Urogynecol J. 2014;25(3):417-424.

10. Zebede S, Smith AL, Plowright LN, Hegde A, Aguilar VC, Davila GW. Obliterative LeFort colpocleisis in a large group of elderly women. Obstet Gynecol. 2013;121(2 Pt 1):279-284.

11. Thubert T, Daché A, Leguilchet T, Benchikh A, Ravery V, Hermieu JF. [Obliterative vaginal surgery for genital prolapse: a retrospective cases series]. Prog Urol. 2012;22(17):1071-1076. French.

12. Lu YX, Hu ML, Wang WY, et al. [Colpocleisis in elderly patients with severe pelvic organ prolapse]. Zhonghua Fu Chan Ke Za Zhi. 2010;45(5): 331-337. Chinese.

13. Agarwala N, HasiakN, Shade M. Graft interposition colpocleisis, perineorrhaphy, and tension-free sling for pelvic organ prolapse and stress urinary incontinence in elderly patients. J Minim Invasive Gynecol. 2007;14(6): 740-745.

14. Ghezzi F, Uccella S, Cromi A, et al. Surgical treatment for pelvic floor disorders in women 75 years or older: a single-center experience. Menopause. 2011;18(3):314-318.

15. Mohammed N, Raschid Hoda M, Fornara P. Prolapse surgery in octogenarians: are we pushing the limits too far? World J Urol. 2013;31(3): 623-628.

16. Lemos NL, Auge AP, Lunardelli JL, Carramão Sda S, Faria AL, Aoki T. Validation of the Pelvic Organ Prolapse Quantification Index (POP-Q-I): a novel interpretation of the POP-Q system for optimization of POP research. Int Urogynecol J Pelvic Floor Dysfunct. 2008; 19(7):995-997.

17. Sung VW, Kaufmann N, Raker CA, Myers DL, Clark MA. Validation of decision-making outcomes for female pelvic floor disorders. Am J Obstet Gynecol. 2008;198(5):575.e1-6.

18. Kaplan PB, Sut N, Sut HK. Validation, cultural adaptation and responsiveness of two pelvic-floor-specific quality-of-life questionnaires, PFDI-20 and PFIQ-7, in a Turkish population. Eur J Obstet Gynecol Reprod Biol. 2012;162(2):229-233.

19. Stark M. The ten step vaginal hysterectomy. Int J Gynaecol Obstet. 2009;107(Suppl 2):S78. Abstract. 
20. Cho MK, Kim CH, Kang WD, Kim JW, Kim SM, Kim YH. Anatomic and functional outcomes with the prolift procedure in elderly women with advanced pelvic organ prolapse who desire uterine preservation. J Minim Invasive Gynecol. 2012;19(3):307-312.

21. Nieminen K, Hiltunen R, Takala T, et al. Outcomes after anterior vaginal wall repair with mesh: a randomized, controlled trial with a 3 year follow-up. Am J Obstet Gynecol. 2010;203(3):235.e1-e235.e8.
22. Hackett NJ, De Oliveira GS, Jain UK, Kim JY. ASA class is a reliable independent predictor of medical complications and mortality following surgery. Int J Surg. 2015;18:184-190.

\section{Publish your work in this journal}

Clinical Interventions in Aging is an international, peer-reviewed journal focusing on evidence-based reports on the value or lack thereof of treatments intended to prevent or delay the onset of maladaptive correlates of aging in human beings. This journal is indexed on PubMed Central, MedLine,

\section{Dovepress}

CAS, Scopus and the Elsevier Bibliographic databases. The manuscript management system is completely online and includes a very quick and fair peer-review system, which is all easy to use. Visit http://www.dovepress. $\mathrm{com} /$ testimonials.php to read real quotes from published authors. 